\section{Improving patient safety through education: how visual recognition skills may reduce medication errors on surgical wards}

\author{
Christopher R. Davis, ${ }^{1}$ Edward C. Toll, \\ Paul M. Bevis, ${ }^{3}$ Helena P. Burden ${ }^{4}$ \\ 'Department of Plastic Surgery, Guy's \\ and St Thomas' NHS Trust, London; \\ 2Department of ENT Surgery, Southmead \\ Hospital, Bristol; ${ }^{3}$ Department of \\ Vascular Surgery, Southmead Hospital, \\ Bristol; ${ }^{4}$ Department of Urology, Royal \\ United Hospital, Bath, UK
}

\section{Abstract}

Medication errors compromise patient safety and cost $\$ 500 \mathrm{~m}$ per annum in the UK. Patients who forget the name of their medication may describe the appearance to the doctor. Nurses use recognition skills to assist in safe administration of medications. This study quantifies healthcare professionals' accuracy in visually identifying medications. Members of the multidisciplinary team were asked to identify five commonly prescribed medications. Mean recognition rate (MRR) was defined as the percentage of correct responses. Dunn's multiple comparison tests quantified inter-professional variation. Fifty-six participants completed the study (93\% response rate). MRRs were: pharmacists 61\%; nurses $35 \%$; doctors 19\%; physiotherapists $11 \%$. Pharmacists' MRR were significantly higher than both doctors and physiotherapists $(\mathrm{P}<0.001)$. Nurses' MRR was statistically comparable to pharmacists $(\mathrm{P}>0.05)$. The majority of healthcare professionals cannot accurately identify commonly prescribed medications on direct visualization. By increasing access to medication identification resources and improving undergraduate education and postgraduate training for all healthcare professionals, errors may be reduced and patient safety improved.

\section{Introduction}

Medication errors affect patients, healthcare professionals and hospital institutions worldwide. The cost incurred for the UK National Health Service (NHS) is estimated at $£ 500$ million per annum with an average of eight additional inpatient days from a single medication error. ${ }^{1}$ Correct prescribing, dis- pensing and administration of medications are vital to reduce the risk of medication errors, promote patient safety and maximize the quality of care patients receive.

Medication errors in hospital may occur at any stage of a patient's journey from assessment to discharge in both elective and emergency situations. An accurate pre-admission medication list assists patient assessment and management in a safe and accurate manner and may reduce initial prescribing errors. Given that $72 \%$ of unintentional medication errors with potential for harm occur when taking the initial medication history, this admissions stage is particularly important in order to reduce prescribing errors. ${ }^{2}$ However, patients are often unable to remember names of their medications and frequently attempt to describe the visual appearance of the preparation or administration device in the hope that the admitting doctor or nurse may recognize their medication by descriptive means. For example, patients commonly refer to blue or brown inhalers when discussing salbutamol and beclomethasone inhalers, respectively.

This practice extends to the description of tablet medication, whereby if a tablet is distinctive in color or shape, patients may offer this information if they cannot recall the name of the drug. Patients may also present with multi-compartment compliance aids, or dosset boxes, containing their medication. The prescription data may not always be enclosed with the box. In emergency situations, when no further information is obtainable, especially in cases of suspected medication overdose, an awareness of how to obtain information relating to the appearance of commonly prescribed generic medications may improve patient safety and reduce iatrogenic morbidity and mortality. Current evidence suggests that medication with a similar appearance, particularly regarding packaging or ampoule design, have been shown to be the cause of near fatal medication errors. ${ }^{3,4}$

Healthcare professionals may use patients' visual descriptions of drug preparations as clues in the medication history to influence drug prescribing or administering. Currently, $60.9 \%$ of medication errors in hospital have been shown to involve administration of the wrong drug ${ }^{5}$ and the risk of errors increases if there are interruptions to the drug administration rounds, resulting in a further $7.5 \%$ of drug administration errors. ${ }^{6}$ Though this extra visual detail may improve the accuracy of prescribing, it is unclear how accurate healthcare professionals are at recognizing descriptions of medications by patients. There is a risk, therefore, that prescribing errors could occur if doctors are unaware of the appearance of medications. Nurses may use visual recognition skills for identifying medications when dispensing or administering drugs. This may occur during
Correspondence: Christopher R. Davis, Department of Plastic Surgery, St Thomas Hospital, London, SE1 7EH, UK.

Tel: +44.207.1887.188.

E-mail: chrisdavis959@hotmail.com

Key words: safety, errors, nursing, medication, prescription, recognition.

Contributions: CRD, ECT, PMB, HPB, study design and manuscript preparation; CRD, ECT, data collection; CRD data analysis.

Conflict of interest: the authors report no conflicts of interest.

Acknowledgments: the authors would like to thank Jessica Bisset at the Research and Innovation Department, University Hospitals Bristol NHS Trust, for generously supporting this research.

Received for publication: 26 September 2011 Revision received: 16 December 2011.

Accepted for publication: 18 December 2011.

This work is licensed under a Creative Commons Attribution NonCommercial 3.0 License (CC BYNC 3.0).

(C) Copyright C.R. Davis et al., 2012

Licensee PAGEPress, Italy

Nursing Reports 2012; 2:e1

doi:10.4081/nursrep.2012.e1

busy rounds, to allow the nurses to doublecheck the medications once they have been removed from the packaging, before administering to patients and to double-check the contents of the container of medications administered to a patient. Certainly, relying on recognition skills alone could harm patients. However, a skilled and accurate appreciation of commonly prescribed medications may reduce drug errors and improve patient safety.

This is the first study to quantify the accuracy of healthcare professionals' visual recognition skills of commonly prescribed medications. The primary outcome measure is to quantify the ability of nurses and doctors to visually identify medications in comparison with other healthcare professionals.

\section{Materials and Methods}

An initial survey was undertaken to outline the proportion of doctors that have used a visual description of a medication to assist in taking a medication history. Twenty-three junior doctors in postgraduate years 3 and 4 working within different hospitals were engaged in a focus group to discuss previous prescribing practices. 
Multidisciplinary team (MDT) members were recruited into the main study. Every nurse, doctor, pharmacist and physiotherapist working on one day within the surgical directorate of a single institution was invited to enter the study. These categories of healthcare professionals were selected due to their individual roles within the medication pathway: prescribing (doctors and nurses); dispensing (pharmacists); administering (nurses). Physiotherapists were recruited into the study to act as a control group given their limited contact with oral medications. Potential study participants were invited to enter the study after the process of identifying medications was described. This approach was conducted within the hospital setting at the pharmacy, ward and physiotherapy department, where every healthcare professional present and eligible was invited.

Inclusion criteria were healthcare professionals that fulfilled the particular MDT roles specified above. All MDT members recruited into the study were fully qualified healthcare professionals. Each staff member was included into the study regardless of seniority, experience or previous training in order to generate a realistic and pragmatic data set using current staff members. Exclusion criteria included MDT members who were actively engaged with patients during the data collection period and student healthcare workers. Participants were only entered once into the study, and were not permitted a second attempt at visual recognition of the medications on any other occasion.

Five commonly prescribed oral medications were placed into a drug-dispensing dosset box shown to each study participant (Figure 1). The medications included omeprazole $20 \mathrm{mg}$ (Losec, AstraZeneca), ciprofloxacin 250mg (generic), bisoprolol 2.5mg (Cardicor, Merck), prednisolone $5 \mathrm{mg}$ (generic), and aspirin $75 \mathrm{mg}$ (generic). These drugs were selected as they are commonly prescribed on medical and surgical wards and include examples of visually distinctive tablets and capsules. The participants were asked to identify the generic name of each drug. No access was given to formularies, databases or discussion with colleagues.

Full approval was granted by the institution (i.e. the corresponding NHS Foundation Trust) to conduct this study before it started.

\section{Statistical analysis}

The mean recognition rate (MRR) was calculated for each MDT group, corresponding to the average number of correct responses expressed as a percentage. A KolmogorovSmirnov assessment of distribution was performed for data from each MDT group and a subsequent one-way analysis of variance (ANOVA) was used for statistical comparison. Inter-professional comparisons were made

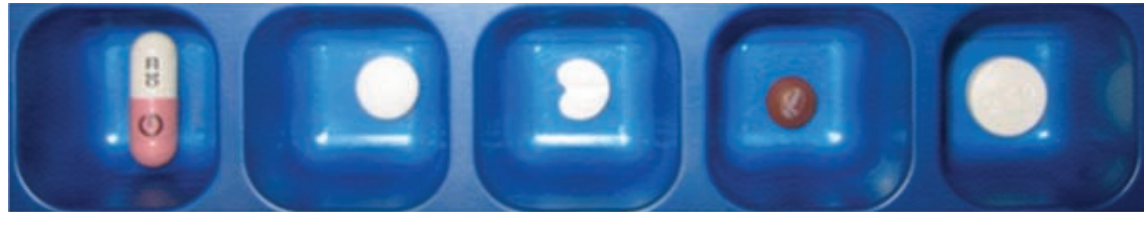

Figure 1. Medication used during study [From left: omeprazole 20mg (Losec, AstraZeneca); aspirin $75 \mathrm{mg}$ (generic); bisoprolol 2.5mg (Cardicor, Merck); prednisolone $5 \mathrm{mg}$ (generic); ciprofloxacin $250 \mathrm{mg}$ (generic)].

using Dunn's multiple comparison tests. Statistical significance was defined as $\mathrm{P}<0.01$. A power calculation was not performed to quantify sample size given the paucity of literature in this area.

\section{Results}

In the initial survey, 8 (34.8\%) of the junior doctors consulted stated that at some point during their careers they had used information from a visual description of a medication as a clue when taking the initial medication history. Sixty MDT participants were invited, with 56 recruited to take part in the study (i.e. $93 \%$ response rate). The participants from each of the healthcare disciplines included: 16 nursing staff (28.6\%), 14 doctors (25\%), 15 pharmacists (26.8\%) and 11 physiotherapists (19.6\%).

The mean MRR for all persons in the study was $33 \%$. Pharmacists were the most successful at identifying medications, with the highest MRR at $61 \%$. Nurses were the second most successful group at identifying medications, with an MRR of 35\%. Doctors had an MRR of $19 \%$, whilst physiotherapists had the lowest MRR at $11 \%$ (Figure 2). The only profession correctly able to identify all five pills were the pharmacists $(n=2)$. There were significant inter-professional differences in the MRR $(\mathrm{P}<0.0001$; Kruskal-Wallis statistic 25.7). Specifically, pharmacists scored significantly higher in their mean recognition rate than both doctors $(\mathrm{P}<0.001)$ and physiotherapists $(\mathrm{P}<0.001)$. However, the ability of nurses to identify medications was statistically comparable to pharmacists $(\mathrm{P}>0.05)$ (Figure 2).

\section{Discussion}

This study has shown that the majority of healthcare professionals are unable to accurately identify commonly prescribed medications. Attempting to use skills of visual recognition could therefore have serious consequences and jeopardize patient safety. Despite this, a third of doctors have used visual descriptions of medications from patients to assist in taking a medication history.

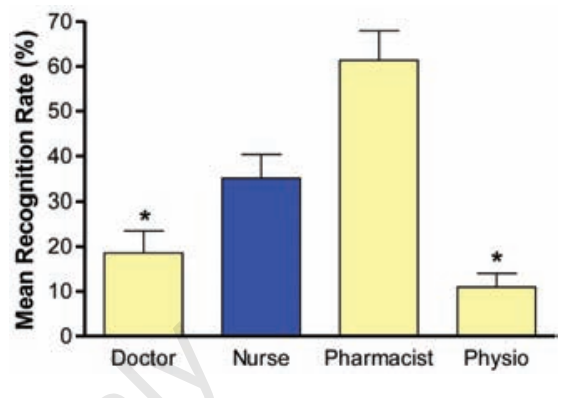

Figure 2. Mean recognition rate+SEM for each category of healthcare professional. ${ }^{*} \mathbf{P}<0.01$ compared to pharmacist; $S E M=S t a n d a r d$ Error of the Mean.

Drug history is of fundamental importance in taking an accurate patient history. Where medical notes or prescription details are unavailable, verbal accounts from patients might be the only source of information when clarifying a drug history. In these situations, patients often volunteer information on the visual appearance of their medications. It would be unprofessional and dangerous to prescribe a medication based solely on a visual description, which could compromise patient safety. Relying purely on visual identification of medications in drug dispensing and administration rounds could also have life-threatening consequences, thus we would discourage such practice. However, there are some situations in which visual descriptions of medications or knowledge of the visual appearance may be the only information available. These include identifying unknown medications stored in dosset boxes, deciphering which medications have or have not been taken by inpatients after drug rounds and in cases of suspected overdose or abuse of medications. In these situations, access to information regarding the visual appearance of medications could improve patient safety. Such information could also prove valuable when assessing patients suspected to be non-compliant with their medications. Medicines that have similar appearances are known to lead to medication errors, sometimes with severe consequences. A case of the administration of intravenous potassium chloride solution instead of sodium bicarbonate has been reported, the reason for this near fatal medication error being the almost identi- 
cal appearance of the ampoules and label design. ${ }^{3}$ Additionally, similarly named or packaged chemotherapeutic agents have the potential for error and subsequent patient morbidity. ${ }^{4}$ The US Joint Commission National Patient Safety Goals for 2005 require organizations to identify and annually review a list of look-alike sound-alike drugs as a basis for safety strategies of avoiding medication errors involving these preparations with similar textural or verbal names. ${ }^{7}$ One such strategy proposed by Friedman includes the education of patients and carers in the home setting to report any changes in the appearance of medications (e.g. size, shape, smell) to healthcare professionals. ${ }^{8}$

It is proposed that widespread access to information and resources regarding the visual recognition of medication could also help to reduce the number of potential errors in drug prescribing, dispensing and administration. This could be achieved by incorporating this theme into undergraduate curricula for medical, nursing and pharmacy undergraduates. Teaching visual identification of various distinctive and commonly used medications into undergraduate and postgraduate medical, nursing and pharmacy curricula may reduce medication errors. Indeed, the Department of Health document, Building a safer NHS for patients - improving medication safety ${ }^{9}$ states that it is now imperative that undergraduate education emphasizes the issue of medication safety. Further studies support the premise of a greater prominence of patient safety themes within nursing curricula ${ }^{10}$ and to target undergraduate nursing teaching to increase confidence in drug administration. ${ }^{11}$ The General Medical Council released a document in December 2009 on prescribing errors relating to education and training. ${ }^{12}$ It highlighted a drug error rate of $8.9 \%$, with half of the healthcare professionals in the study raising concerns of dissatisfaction with their training in this area. This further strengthens the need for improving medication training for all healthcare professionals at all levels.

There are a number of limitations to this study. Methodologically, no power calculation was made before starting the study, and as such a sample size calculation was not performed. However, the reason for this was due to the paucity of literature relating to previous studies in this area. We, therefore, aimed to maximize recruitment into this study, and would envisage that the study sample sizes obtained of 23 for the preliminary study and 56 for the main study provide reliable data and new evidence to the limited evidence-base that currently exists. Further limitations include the potential omission of additional specialities within the MDT, absent hospital staff during the study period, and a finite number of medications selected for the study. However, the snap-shot approach to the study recruit- ment enabled a representative assessment of the everyday workings of tertiary hospital care to be made, and as such the data are more likely to represent regular clinical activity in healthcare professionals. Furthermore, the medications were selected on the basis of frequent patient usage, readily identifiable features, and common to different specialities. The data concerning medication identification may, therefore, be of broader interest to a range of medical and surgical specialities. All authors are medical staff.

Clinical practice might include early liaison with primary care and utilization of the pharmacy service in confirming the accurate drug history in order to reduce medication errors. In 2009, Valsieff et al. found that prescribing errors in the emergency department were reduced by pharmacists eliciting prompt and accurate medication histories and preparing prescription charts at the earliest opportunity. ${ }^{13}$ In addition, the availability, accessibility and breadth of resources that aid visual identification of medications should be improved to assist multidisciplinary team members in the processes of taking a medication history, prescribing, dispensing and administration of drugs. Pre-existing resources available include websites featuring tablet identifcation programmes designed to aid healthcare professionals. These websites generally include parameters of shape, color and inscription, which the user can input from a drop-down list. Such sites contain photographic databases of oral medications that fit the inputted description. The use of these tools and databases in the setting of the Accident and Emergency Department or Assessment Unit could aid the process of accurate medication history taking if no other data are available, thereby reducing the potential for medication errors and improving patient management. Such material might be collected and managed by personal digital assistant (PDA) technology, which has been shown to increase the accuracy and speed of medication administration by nurses. ${ }^{14}$

\section{Conclusions}

Visual recognition of medications amongst all members of the MDT is poor, and relying on this information for completing medication history and prescribing regular medications could lead to medication errors. However, in certain situations there may be no other relevant information available, and access to reliable information of the visual appearance of medications may prove useful or even life-saving. We believe that knowledge of and access to resources for visually identifying medications should be improved in an effort to reduce prescribing errors.

\section{References}

1. Audit Commission. A spoonful of sugarimproving medicines management in hospitals. London: Audit Commission; 2001.

2. Pippins JR, Ghandi TK, Hamann C, et al. Classifying and predicting errors of inpatient medication reconciliation. J Gen Intern Med 2008;23:1414-122.

3. Shah HD, Shah M. A case of look-alike medication errors. Indian $\mathrm{J}$ Pharmacol 2011;43:482-3.

4. Schulmeister L. Look-alike, sound-alike oncology medications. Clin J Oncol Nurs 2006;10:35-41.

5. Kuitunen T, Kuisma P, Hoppu K. Medication errors made by health care professionals. Analysis of the Finnish Poison Information Centre data between 2000 and 2007. Eur J Clin Pharmacol 2008;64:769-74.

6. Pham JC, Story JL, Hicks RW, et al. National study on the frequency, types, causes, and consequences of voluntarily reported emergency department medication errors. J Emerg Med 2011;40:485-92.

7. McCoy LK. Look-alike, sound-alike drugs review: include look-alike packaging as an additional safety check. Jt Comm J Qual Patient Saf 2005;31:47-53.

8. Friedman MM. Medication safety: lookalike/sound-alike drugs in home care. Home Healthc Nurse 2005;23:243-53.

9. Department of Health. Building a safer NHS for patients - improving medication safety. London: Department of Health, 2004.

10. Wakefield A, Attree M, Braidman I, et al. Patient safety: do nursing and medical curricula address this theme? Nurse Educ Today 2005;25:333-40.

11. Banning M. Nurse prescribing, nurse education and related research in the United Kingdom: a review of the literature. Nurse Educ Today 2004;24:420-7.

12. Dornan T, Ashcroft D, Heathfield H, et al. An in-depth investigation into causes of prescribing errors by foundation trainees in relation to their medical education: EQUIP study. General Medical Council, University of Manchester; 2009.

13. Valisef HM, Whitten LE, Pink JA, et al. The effect on medication errors of pharmacists charting medication in an emergency department. Pharm World Sci 2009; 31:373-9.

14. Greenfield S. Medication error reduction and the use of PDA technology. J Nurs Educ 2007;46:127-31. 